\title{
RIEMANN SURFACES OF CLASS A
}

\author{
BY \\ ROBERT OSSERMAN( ${ }^{(1)}$
}

This paper contains results of a somewhat varied nature, all obtained from a detailed examination of a special class of surfaces. These results include a method for the isometric embedding of Riemann surfaces in space, an application of this method for the construction of a hyperbolic surface in space, and several criteria in the problem of type.

The first three sections are devoted to developing the necessary preliminary material. In $\$ 1$ we give an intuitive picture of the embedding process, and also the exact definitions to be used throughout the paper. $\$ 2$ contains a brief discussion of the surfaces to be considered, simply-connected covering surfaces of the plane having only algebraic singularities over finite points, which are denoted as surfaces of class A. A method is given for subdividing these surfaces into sheets, this subdivision being the main tool for the embedding. In $\$ 3$ we define and study the Euclidean metric on the surface.

The embedding itself is carried out in $\$ 4$, where we show how a large class of Riemann covering surfaces can be represented isometrically as nonselfintersecting surfaces in 3-space. The interest in the embedding method is partly theoretical, since the existence of such an embedding is somewhat surprising in itself, and partly practical, in the sense that by viewing a surface in the imbedded form one often obtains a much better intuitive picture of its internal structure.

The author has indicated at the 1954 International Congress [8] how this embedding method could be used to settle a question on the existence of certain hyperbolic surfaces in 3-space. The details are carried out in $\$ 5$ with slight refinements so that the surface obtained is everywhere infinitely differentiable. The method used here for removing the singular points may be used in conjunction with the author's paper [9] to provide a different way of settling the original question.

Finally, we turn to the problem of type for surfaces of class A. These surfaces were originally considered by Ahlfors [1] who introduced the function $n(t)$ as a measure of the branching and found a sufficient condition for parabolic type. $\$ 6$ contains a number of new results on the use of the function $n(t)$ in the determination of type.

Presented to the Society, September 2, 1955 under the title On Ahlfors' counting function $n(t)$; received by the editors August 22, 1955.

(1) This paper is based on one part of the author's thesis at Harvard University, April, 1955. The work was supported in part by the Office of Naval Research on contract N5ori-07634. 
The author wishes to thank Professor Ahlfors for his guidance and stimulation throughout this investigation, and also Mr. Henry Landau for many valuable conversations.

1. Introduction. In this section we give first some intuitive notions about the material to follow, and then state the precise definitions and notation which will be used throughout the paper.

1.1. We start with a simple example. We are given the Riemann surface of $w^{1 / 2}$ and wish to embed it isometrically as an ordinary surface in 3-space. To do so we consider the surface in the usual fashion as consisting of two sheets, each a copy of the $w$-plane slit along the positive real axis, which are joined by crosswise identification. What we shall do is simply embed each sheet separately in $x, y, z$-space in such a manner that the edges to be identified coincide. Roughly speaking, this is done by folding up each sheet fanwise around the origin and then matching the edges. More precisely, choose one of the two sheets and divide it up into eight $45^{\circ}$ sectors with vertex at the origin. Denote these by $A_{1}, \cdots, A_{8}$ in order of increasing argument starting with the positive real axis. Let $L_{1}, \ldots, L_{9}$ be the edges of these sectors in the same order, where $L_{1}$ and $L_{9}$ lie on opposite edges of the slit along the positive real axis. We now place sector $A_{1}$ in $x, y, z$-space in such a manner that the edge $L_{1}$ coincides with the positive $x$-axis, and the whole sector projects onto a $22 \frac{1}{2}^{\circ}$ sector in the $x, y$-plane. We then place the sector $A_{2}$ so that its edge $L_{2}$ coincides with the edge $L_{2}$ of $A_{1}$, and so that the edge $L_{3}$ lies in the $x, y$-plane. Then $A_{2}$ will also project onto a $22 \frac{1}{2}^{\circ}$ sector, and we may continue the process, placing each of the sectors so that their common edges coincide and so that they each project onto a $22 \frac{1}{2}^{\circ}$ sector. The edge $L_{9}$ of $A_{8}$ will then lie along the negative $x$-axis and all eight sectors together will project onto the upper half of the $x, y$-plane. The same procedure can be applied to the other sheet of the surface so that it projects onto the lower half plane. We now observe that the pair of edges lying along the positive $x$-axis and the pair lying along the negative $x$-axis are precisely those which were to be identified in the original surface. We may therefore glue the sheets back together along these edges and obtain a nonselfintersecting surface which was derived from our original surface by a succession of foldings, and which is therefore an isometric image of that surface with all inner properties left invariant.

1.2. The main purpose of the next few sections is to show how this embedding process may be carried out rigorously for a large class of simplyconnected surfaces. The idea is simply to divide the surface into sheets in such a way that each sheet may be folded up fanwise in the above manner. This allows us to place an isometric image of the sheet in $x, y, z$-space in such a way that it projects onto an arbitrarily small sector of the $x, y$-plane. If this intuitive picture is kept in mind it may help in following the details of the decomposition carried out in the next section. 
The main application of this method is to the problem treated in $\S 4$. The question is to prove the existence of a hyperbolic surface in 3-space satisfying certain conditions. Rather than start with a surface and prove that it is hyperbolic, our method is to start with a Riemann surface known to be hyperbolic and re-embed it in 3-space.

1.3. There is one other point which may be worth mentioning here in connection with our method of embedding. It is clear that the surfaces of $w^{1 / n}$ can be treated in a manner similar to that of $w^{1 / 2}$, since we can make each sheet project onto a sector of angle $2 \pi / n$ in the $x, y$-plane. However, it is also interesting that we may use this method to embed the surface of $\log w$. We need only start with an arbitrary sheet and fold it so that it projects onto an angle of $\pi / 4$. The sheets immediately "above" and "below" may then be placed on either side so that they each project onto an angle of $\pi / 16$. The next two are made to project onto angles of $\pi / 32$, and so on. The total surface will then project onto a quarter plane, and it is clear that by choosing different angles we could have made the total surface project onto an arbitrary sector. The ideal boundary of the surface corresponds to the edges of this sector, and the logarithmic branch point at the origin corresponds to the vertex. This is not interesting from the conformal point of view in which the ideal boundary is a single point, but it may be found useful in giving a clear intuitive picture of why a logarithmic branch point cannot be considered an interior point of the surface. It seems that a number of beginning students of Riemann surfaces have a great deal of difficulty in grasping this point.

1.4. We now proceed to give the exact definitions and notation which will be used throughout this paper.

We shall use the standard Weyl-Rado definition of a Riemann surface as a two (real) dimensional manifold with a complex analytic structure. For the most part our interest will be centered on those Riemann surfaces which are also covering surfaces of the plane. For the sake of uniformity we shall assume throughout that they are covering surfaces of the complex $w$-plane. By this we shall mean that there is associated with the surface a nonconstant analytic function $w=\pi(p)$ from the surface into the $w$-plane. The function $\pi$ is called the projection function. We may speak of the derivative of an analytic function on a Riemann surface with respect to a given coordinate system, and the points where the derivative $\pi^{\prime}$ is zero are independent of the choice of coordinate system. These points are called the branch points of the surface. All other points of the surface have a neighborhood in which the projection mapping is one-one. An arc $C^{*}$ on the surface is called a continuation of the surface along an arc $C$ in the plane, if $C$ can be written as $\pi(p(t))$, where $C^{*}$ is described by $p(t)$. Once $C$ and $p(0)$ are fixed, $C^{*}$ is uniquely determined unless it passes through a branch point. At a branch point of order $n$ there are exactly $n$ possible continuations. (For further details connected with these definitions see [3, Chapter IV].) 
1.5. In all that follows we shall be interested only in simply-connected Riemann surfaces. These arise from the classical theory through the consideration of the inverses of single-valued functions. We shall use the following precise definition. Let $w=f(z)$ be a single-valued function whose domain of analyticity is a simply-connected region $\Omega$. We associate to the inverse function $z=g(w)$ a Riemann surface whose points are the set of ordered pairs $(w, z)$ such that $w=f(z)$. As neighborhood patches we choose the sets of points $(w, z)$ whose $z$-values fill out an open disk in $\Omega$. The analytic structure is obtained by assigning to each coordinate patch the corresponding disk under the mapping $(w, z) \rightarrow z$. The projection function $(w, z) \rightarrow w$ then makes this Riemann surface a covering surface of the $w$-plane. (Classically one pictures the surface to be spread out over the w-plane in such a manner that all points $(w, z)$ with the same $w$-coordinate lie over the point with that coordinate in the w-plane.) The surface so constructed is called the Riemann surface of the function $g(w)$.

1.6. Suppose now we start with a given simply-connected open Riemann surface $W$ which is a covering surface of the $w$-plane. By the Koebe uniformization theorem we can find a function $z=\phi(p)$ mapping the surface one-one conformally onto a simply-connected region $\Omega$ of the $z$-plane. If we denote its inverse by $p=\psi(z)$, then the composed mapping $w=f(z)=\pi \psi(z)$ is singlevalued in $\Omega$. In general $f(z)$ can be continued analytically outside of $\Omega$, but in the case that $\Omega$ is the precise region of analyticity of $f(z)$, we can form the Riemann surface $W^{\prime}$ of the inverse function $z=g(w)$ as above. Since both $W$ and $W^{\prime}$ are conformally equivalent to $\Omega$, we can form the composed mapping $p \rightarrow(w, z)$, where $z=\phi(p)$ and $w=f(z)$, which is a one-one conformal mapping of $W$ onto $W^{\prime}$ preserving the projection function. Thus, in this case the original surface $W$ may itself be considered the Riemann surface of the function $g(w)$.

We may point out here that a given Riemann surface can be spoken of as the Riemann surface of a function only if it is given as a covering surface.

1.7. We now note that by the Riemann mapping theorem the region $\Omega$ in the $z$-plane may be chosen to be either the whole plane or the interior of the unit circle. The surface is then called of parabolic or hyperbolic type, respectively. In the original problem of type one was given the Riemann surface as a covering surface, and required to determine the type in terms of the distribution of branch points or other structural properties of the surface. In $\$ 6$ we are concerned specifically with this problem. The problem of type, however, applies also to Riemann surfaces which are given in other ways, and in $\$ 5$ we consider a case where the points of the surface are those of an ordinary surface in 3-space and the conformal structure is determined by the Euclidean metric.

There is one final remark which should be made at this point. The interest in the original problem of type was derived largely from the fact that the sur- 
faces considered were those of the inverses of either entire functions or functions analytic in the unit circle. It was hoped that the classification of these surfaces would lead to information concerning the corresponding classes of functions. In actual fact, however, this procedure was reversed, and most of the criteria of type were derived from theorems about single-valued functions, such as Picard's, Bloch's, and Nevanlinna's. The paper of Ahlfors [1] represents one of the first attempts to derive information directly from the structure of the surface, and it is in this direction that we attack the problem in $\S 2, \S 3$, and $\S 6$.

\section{The Class A. Canonical subdivision.}

2.1. Definition. A Riemann surface will be called of class $\mathrm{A}$ if it is a simply-connected covering surface of the finite plane, and if all continuations of the surface along an arbitrary arc which ends at a finite point of the plane lead to an interior point of the surface.

Thus, a simply-connected covering surface is of class A if it has no boundary points or nonalgebraic singularities over finite points.

If we are given a simply-connected covering surface $W$ over the w-plane, and assign to it the single-valued function $w=f(z)$ as in 1.6 , then the condition that $W$ be of class $\mathrm{A}$ is that $f(z)$ have no finite asymptotic values. Namely, an asymptotic value is the end point of the projection of a path on the surface which corresponds to a path in the $z$-plane going to the boundary. Since the end point of such a path cannot be an interior point of the surface, our definition precisely precludes its projection from being finite. Furthermore, in the case of a Riemann surface of class $A$, the region $\Omega$ onto which it is mapped is exactly the region of analyticity of $f(z)$. Namely, if one could extend $f(z)$ to be even continuous at some point of the boundary of $\Omega$, then a path leading to that point would produce a finite asymptotic value. Thus, by the reasoning of 1.6 , we see that the surface $W$ may be identified with the surface of the inverse function to $f(z)$. In other words, the Riemann surfaces of class A are precisely the Riemann surfaces of the inverses to functions which are single-valued and have no finite asymptotic values.

Some simple examples of functions whose inverses have Riemann surfaces of class $\mathrm{A}$ are polynomials, sine and cosine, and the Schwarz triangle functions. These last include cases of both parabolic and hyperbolic surfaces.

2.2. We introduce now a method of studying the structure of an arbitrary surface of class A by means of a canonical dissection into sheets.

A sheet is constructed as follows. Starting with a copy of the w-plane, a number of rays are removed, all the rays being radial with respect to some given point which we shall call the center of the sheet. We require that only a finite number of rays shall intersect any finite circle. Thus, in particular, there are only a countable number of rays removed on each sheet. The points remaining in the plane after the removal of these rays will be called interior points of the sheet. We now add boundary points as follows. Let $L$ be one of 
the rays which was removed. We assign two copies of $L$ to the surface, denoting them by $M$ and $N$. As a neighborhood base for points of $M$ we choose closed semicircles with diameter on $M$. These semicircles must not intersect any of the other removed rays, and they must all lie on the side of $L$ determined by the condition that each semicircle is intersected by all rays obtained by clockwise rotation of $L$ through a sufficiently small angle about its end point. For neighborhoods of a point on $N$ we choose the closed semicircles with diameter on $N$, lying on the opposite side of $L$. $M$ and $N$ together will be called a slit of the sheet, and they will be denoted separately as the edges of the slit.

In what follows we shall represent an arbitrary Riemann surface of class $\mathrm{A}$ as the union of sheets of the above type, with certain identifications. Every sheet of the surface, except for at most one, will have the additional property that one of its slits will have the center of the sheet as end point. We shall refer to this slit as the fundamental slit of the sheet.

2.3. Our procedure will be to show first a specific method for constructing Riemann surfaces of class A by combining sheets in a certain fashion, and then to prove that one actually obtains all Riemann surfaces of class $A$ by this method.

In order to make the procedure clear, we illustrate with an example the sort of construction which must be made.

First of all it should be noted that in order to embed each sheet of a surface it will be necessary later on to fold it up fanwise about a branch point, as was done for $w^{1 / 2}$. Once a sheet is embedded, in order to attach further sheets over common slits, these slits must be made radially with respect to the branch point which is at the vertex of the fan.

Suppose now that we consider as an example the surface which has simple branch points in each sheet over the points $-1,0$, and +1 . If the point -1 is the vertex of a sheet folded into a fan, then to attach the next sheet at 0 , we must make a slit from 0 to infinity, radial with respect to -1 . But such a slit passes through +1 , so that we cannot simply identify the edges of the slit in the two sheets. In fact, we must make this identification from 0 to +1 and leave the rest of the slit free to attach to the following sheet in order to obtain the necessary branch point at +1 . This explains the necessity for introducing the points " $p$ " and " $q$ " in the general construction which follows. We now proceed to the general case.

We start with an arbitrary sheet $W_{0}$. To each slit $L$ on $W_{0}$ we assign a finite number of sheets each of whose fundamental slit coincides with $L$. These will be called the sheets of the first generation. The union of $W_{0}$ and the sheets of the first generation after certain identifications are made will be denoted by $W_{1}$. $W_{1}$ is not necessarily a surface in the strict sense, since it may contain points (on the edges of slits) which have no neighborhoods homeomorphic to an open disk. Such points shall be referred to as boundary points 
and all others as interior points. We shall use the word surface in an extended sense, allowing the existence of boundary points.

To avoid repetition we proceed immediately to the general inductive step which describes how the approximating surface $W_{n}$ is obtained from $W_{n-1}$ by adjoining the sheets of the $n$th generation. We assume that

(a) $W_{n-1}$ is the union of at most a countable number of sheets.

(b) Every point of $W_{n-1}$ has a neighborhood homeomorphic to an open disk, with the following possible exceptions:

1. those points lying on slits other than the fundamental one on sheets of the $(n-1)$ st generation;

2 . those points lying past a given point $p$ which may have been distinguished on one edge of the fundamental slit on a sheet of the $(n-1)$ st generation;

3. those points lying past a point $q$ on one edge of a slit of a former generation if the point $q$ has been identified with some point $p$ of the type mentioned in 2.

(c) The points $p$ must have been chosen so that every one is identified either with a point $q$, or else with another point $p$ lying on the opposite edge of the fundamental slit of another sheet of the $(n-1)$ st generation.

(d) Pairs of identified points, when considered as complex numbers in the plane, must be equal.

Under these conditions we form the surface $W_{n}$ and show that it has all the same properties.

First of all, to each slit other than the fundamental one on sheets of the $(n-1)$ st generation we assign a finite number of sheets of the $n$th generation, all having the given slit as fundamental slit. Let the given slit have edges $M$ and $N$, and let the fundamental slits on the new sheets have edges $M_{i}$ and $N_{i}, i=1,2, \cdots, m$. We either identify all points of $M$ with the corresponding points of $N_{1}$, or else we make the identification only up to a given point $p$. Similarly, for each $i, i=2, \cdots, m$, we identify either all of $M_{i-1}$ with all of $N_{i}$ or else make the identification only up to a given point. The same thing is done with $M_{m}$ and $N$.

We now assign neighborhoods to the points obtained by identification. A pair of identified points lying in the interior of an identified segment will have as neighborhood basis all sufficiently small circles about them, each consisting of the union of two semicircles whose common diameters have been identified. Under the given identification the end points of all the $m+1$ slits involved will become a single point $p_{0}$. A neighborhood in the neighborhood base for $p_{0}$ is the union of $m+1$ sufficiently small equal circles around $p_{0}$, one from each of the sheets containing $p_{0}$, where the prescribed identifications have been made along the edges of slits. One sees immediately that each such neighborhood is homeomorphic to an open disk, by mapping the $m+1$ slit circles by successive branches of $\left(w-w_{0}\right)^{1 /(m+1)}$, where $w_{0}$ is the complex value of $p_{0}$. 
We now consider those boundary points of $W_{n-1}$ which fall into the categories 2 and 3 of (b). Each of these is associated with a point $p$ on some sheet of the $(n-1)$ st generation. To each such point $p$ we have associated all points past $p$ on an edge of type $M$ on some sheet, and all points past $p$ on an edge of type $N$ of another sheet. These two rays from $p$ do not form the two edges of a slit, since they do not lie on a single sheet. However, if we assign to each point $p$ a finite number of sheets having these two rays as fundamental slit, then the construction given above for attaching these sheets can be carried out without change. The point $p$ becomes an interior point in exactly the same manner as the point $p_{0}$ above. The surface thus obtained will be denoted as $W_{n}$. We see then that all boundary points of $W_{n-1}$ have become interior points unless they fall into one of the categories 2 or 3 of (b). The only other boundary points are the edges of slits on sheets of the $n$th generation. Thus $W_{n}$ satisfies conditions (a), (b), (c), and (d), and the process may be continued inductively.

If after a finite number of steps we arrive at a surface $W_{n}$ having only interior points, then the process is concluded. Otherwise we continue to adjoin new generations of sheets, and the final surface $W$ is the union of all of these. In the latter case we impose the additional requirement

(e) any path consisting of an infinite number of line segments joining together points of type $p$ or $p_{0}$ must have infinite total length.

2.4. We proceed now to prove that $W$ is a Riemann covering surface of the plane.

First of all, we note that we have obtained a true surface. Namely, if the process stops after a finite number of steps, then $W_{n}=W$ has only interior points and there is nothing to prove. In the general case, since $W$ is a union of sheets, an arbitrary point $p_{1}$ of $W$ must lie on some sheet. We need only enumerate the cases to see that it will always be an interior point of $W$. Namely, if it is an interior point of the sheet there is no question. If it is the end point of a slit it becomes a point $p_{0}$ and hence an interior point. The only other possibility is that it lies on one edge of a slit, in which case I claim that it must be either in the interior of an identified segment, or else a point $p$. To see this, let us denote by $L$ the edge of the slit containing $p_{1}$, and let $p_{2}$ be the end point of $L$. A finite number of sheets are adjoined at $p_{2}$, and on one of these there must be a slit having one edge identified with all or part of $L$. If $p_{1}$ is not included in the identified part, then there must be a point $p$ between $p_{1}$ and $p_{2}$. Repeating the reasoning for the part of $L$ past this point $p$ we see that if the point $p_{1}$ is never identified with a point on another edge, then there must be an infinite number of points $p$ between $p_{2}$ and $p_{1}$. But then the path joining these successively will consist of an infinite number of segments having finite total length, which contradicts condition (e). Thus at some point in the construction $p_{1}$ must have been either an interior point or an end point of an interval of identified points, and in both cases it becomes an interior point of $W$. 
To each point of $W$ we have thus assigned a set of neighborhoods, and to each neighborhood an explicit homeomorphism onto an open disk in the complex plane. Since two such disks are related either by the identity map or a root, the structure thus assigned to the surface is analytic, and $W$ thus becomes a Riemann surface.

We next note that $W$ is clearly a covering surface of the $w$-plane, where the projection function is defined by assigning to each point its complex value. This value is uniquely determined, since each point which does not lie on a unique sheet must arise from identification of points on the edges of slits, and this identification was only made between points having the same complex value. By the choice of neighborhood systems on $W$, the projection function is everywhere analytic. Those points which we have previously called points $p$ and $p_{0}$ are the branch points of $W$.

2.5. We now show that $W$ is simply-connected. We note first that if $p$ is an arbitrary branch point of $W$, and if the order of $p$ is $m+1$, then there are $m$ sheets of the same generation which have $p$ as center, and a sheet of some lower generation which contains $p$. Furthermore, on this latter sheet $p$ is not the center. We can therefore assign to an arbitrary point $q$ of $W$, a polygonal path $Q$ leading to the center of $W_{0}$ in the following fashion. If $q$ lies on a unique sheet, draw the line segment from $q$ to the center of the sheet on which it lies. If it lies on more than one sheet choose the sheet of lowest generation. The center of this sheet being a branch point will lie on a sheet of lower generation on which it will not be the center, and we may join it by a line segment to the center of that sheet. Continuing this process we must arrive in a finite number of steps at the center of $W_{0}$. If we are given an arbitrary closed curve on $W$ we can deform it homotopically to the center of $W_{0}$ by allowing each point $g$ on the curve to move uniformly along the path $Q$. More specifically, if the curve is given by $q=q(\theta)$, a continuous map of the unit circumference, then we can extend this map continuously over the interior of the unit circle by mapping the radius of angle $\theta$ by a uniform stretching onto the path $Q(\theta)$ corresponding to $q(\theta)$, with the origin mapping onto the center of $W_{0}$.

We thus see that $W$ satisfies all the conditions for Riemann surfaces of class A, except that it may have a nonalgebraic singularity over a finite point of the plane. This means, in view of the fact that all surfaces of class A will be shown to be representable in the above fashion, that our construction yields a slightly larger class of surfaces. Since it is the representation in sheets in the above manner which is of importance in our later proofs, we see that these proofs, and in particular the embedding theorem, actually hold for the larger class of surfaces. The important fact is that all nonalgebraic singularities be at infinite distance along the surface, and that is guaranteed by condition (e).

2.6. We must now show that every Riemann surface of class $A$ is in fact representable as a union of sheets in the manner described above. To do this, 
let $W$ be an arbitrary Riemann surface of class A, and assign to it, as in 1.6, the function $w=f(z)$, analytic and single-valued in the simply-connected region $\Omega$. Let $W^{\prime}$ be the Riemann surface of the inverse function $z=g(w)$. We have seen in 2.1 that $W$ and $W^{\prime}$ may be identified. We shall now construct a third surface $W^{\prime \prime}$ as a union of sheets, and show that all three surfaces are conformally equivalent.

We start with an arbitrary point $z_{0}$ of $\Omega$ such that $f^{\prime}\left(z_{0}\right) \neq 0$, and let $w_{0}=f\left(z_{0}\right)$. By the single-valuedness of $f(z)$, there is a unique branch of $g(w)$ which assigns the value $z_{0}$ to $w_{0}$. We may continue this branch analytically to the boundary of an arbitrary circle $\left|w-w_{0}\right|<R$ along every radius except for at most a finite number. Namely, if we reached singularities along an infinite number of radii, then these singularities would have a point of accumulation, and a path to this point of accumulation would correspond to a path to the boundary of $\Omega$, in which case $f(z)$ would have a finite asymptotic value. We thus see that this branch of $g(w)$ may be continued radially to infinity in all except for at most a countable number of directions, and that in each of these exceptional directions it must have encountered an algebraic singularity. If we remove the rays from each of these singular points to infinity, we obtain the set of interior points of the sheet $W_{0}$. These interior points form a simply-connected region which is mapped one-one conformally by $f(z)$ onto a simply-connected region $H_{0}$ in $\Omega$, and which is therefore conformally equivalent to the region on $W^{\prime}$ consisting of those points $(w, z)$ with $z$ in $H_{0}$.

We wish now to extend our mapping to a topological map of the whole sheet $W_{0}$ onto $\bar{H}_{0}$, the closure of $H_{0}$ with respect to $\Omega$. We note first of all that by the continuity of $f(z)$, every point of the boundary of $H_{0}$ must map onto a point of one of the slits of $W_{0}$. Since the mapping is locally one-one and conformal except at isolated points, the boundary of $H_{0}$ must consist of piecewise analytic arcs. To each point other than the end point of a removed ray $L$ in the $w$-plane, correspond two distinct prime ends which we may identify with the corresponding points on the edges $M$ and $N$. We can therefore extend the mapping to a one-one map between the boundar ies. To each prime end of $W_{0}$ corresponds a unique prime end of $\bar{H}_{0}$. Furthermore, since the boundary of $H_{0}$ is piecewise analytic, each prime end corresponds to a single point. Finally, we can show that no point of $\bar{H}_{0}$ corresponds to two distinct prime ends. Namely, if this were so, then this point would correspond to two distinct points of $W_{0}$ under the mapping $f(z)$. Since $f(z)$ is single-valued, the two points must lie on opposite edges of a slit. Suppose this were so, and let the slit be $L$ with end point $b$. If $b$ is a branch point of order $m$, then in a neighborhood of $b, g(w)$ maps the edges $M$ and $N$ onto two distinct analytic arcs making an angle of $2 \pi / m$ at $c=g(b)$. Our assumption now is that these two arcs meet at a point $d$, interior to $\Omega$. But they would then bound a compact subregion $C$ of $\Omega$. If we draw an $\operatorname{arc} \gamma$ from $b$ to infinity in the interior 
of $W_{0}$, we can choose a branch of $g(w)$ at $b$ which maps $\gamma$ in a vicinity of $g(b)$ into $C$. Then the image of $\gamma$, since it goes to the boundary of $\Omega$, must cross the boundary of $C$. But the boundary of $C$ consists uniquely of points which map onto the ray $L$, which is a contradiction, proving the impossibility of two distinct points of $W_{0}$ mapping onto the same point of $\Omega$.

We now define the sheets of the first generation. To each slit $L$ on $W_{0}$ whose end point $b$ is a branch point of order $m+1$, we assign $m$ sheets, each with fundamental slit along $L$. The function $g(w)$ has $m+1$ distinct branches in the plane slit along $L$. One of these is the branch which maps the interior of $W_{0}$ onto $H_{0}$. Each of the other branches may be continued radially to infinity from $b$ in all except for at most a countable number of directions, and repeating the same reasoning as before, we see that to each branch we may assign one of the $m$ sheets with suitable slits. Each of these sheets maps onto a simply-connected region of $\Omega$ whose boundary makes an angle of $2 \pi /(m+1)$ at $g(b)$. If we number these regions in counterclockwise order around $g(b)$ and give the corresponding sheets the same order, then we see that the identification of the edges $M_{i}$ and $N_{j}$ of the slit $L$ described in 2.3 will precisely identify points having the same image in the $z$-plane. This image is the analytic arc obtained by continuing a particular one of the branches of $g(w)$ along $L$. If this branch may be continued analytically to infinity, then the entire edges are identified. If we come to a branch point, then the identification is made only up to this point, which then becomes a point $p$.

We thus obtain the surface $W_{1}$, and succeeding generations are adjoined in precisely the same manner. If after a finite number of steps the surface $W_{n}$ maps onto all of $\Omega$, then we let $W_{n}=W^{\prime \prime}$. Otherwise the image of each $W_{n}$ has a relative boundary in $\Omega$ which corresponds to the slits on $W_{n}$ and the process can be continued, until we arrive after a countable number of steps at the surface $W^{\prime \prime}$ which is the union of all the $W_{n}$.

That $W^{\prime \prime}$ must map onto all of $\Omega$ may be seen as follows. Suppose $z_{1}$ were a point of $\Omega$ not contained in the image of any $W_{n}$. Let $\gamma$ be an analytic arc in $\Omega$ joining $z_{0}$ to $z_{1}$. The image of $\gamma$ under $f(z)$ will be an analytic arc $\Gamma$ of finite length starting at $w_{0}$. Let $\Gamma^{*}$ be that continuation of $W^{\prime \prime}$ along $\Gamma$ which maps into $\gamma$. Since $\Gamma^{*}$ is not contained in any of the $W_{n}$, we can define for each $n$, the point $p_{n}$ as the last point of $\Gamma^{*}$ on a sheet of the $n$th generation, and $S_{n}$ as the corresponding sheet. The point $p_{n}$ is on a slit $L_{n}$, and all the sheets of the $(n+1)$ st generation which the part of $\Gamma^{*}$ after $p_{n}$ can intersect must have as center the end point of $L_{n}$. In particular, the center $c_{n+1}$ of $S_{n+1}$ must be in $S_{n}$ for each $n$, so that if we denote by $Q_{n}$ the polygonal path joining successive centers from $c_{n}$ down to $c_{0}$, the length of $Q_{n}$ will be less than the length of the part of $\Gamma^{*}$ up to $p_{n}$, and hence less than the total length of $\Gamma^{*}$. (This is proved in 3.2.) Since this is true for all $n$, the length of the polygonal path joining all $c_{n}$ successively is also less than the length of $\Gamma^{*}$. We have thus a path on $W^{\prime \prime}$ which has finite length and joins an infinite 
number of branch points. If its image in the $z$-plane went to the boundary of $\Omega$ we would have an asymptotic value of $f(z)$, and if not we would have a nonalgebraic singularity of $W$. Since both cases are impossible, we have proved that $W^{\prime \prime}$ maps one-one onto all of $\Omega$. By the conformal structure defined on surfaces made of sheets, we see that this correspondence is conformal.

We thus have a one-one conformal map of each of the surfaces $W, W^{\prime}$, $W^{\prime \prime}$, onto $\Omega$ with the property that if a point on the surface corresponds to a point $z$ in $\Omega$, then its projection will be $w=f(z)$. Thus, by composition we get a one-one conformal mapping between each pair of surfaces, always preserving the projection function. We may therefore identify the surface $W^{\prime \prime}$ with each of the other two, and thus obtain a decomposition of the original surface into sheets.

3. The Euclidean metric on the surface. The purpose of this section is to define what we mean by the Euclidean metric on a Riemann surface of class $\mathrm{A}$, and to investigate some of its properties. We shall need these results mainly for $\S 6$.

3.1. Given an arbitrary curve on the surface as the continuous image of the unit interval, we know that as a compact set it can intersect only a finite number of sheets and slits. If the metric in each sheet is taken to be that of the $w$-plane of which it is a copy, and if the part of the curve lying in the interior of each sheet has a well-defined finite length, and also the part lying on each slit has a well-defined finite length, then we define the total length of the curve to be the sum of these. Otherwise we assign the curve infinite length.

We see immediately that the length of any curve on the surface is greater than or equal to the length of its projection in the $w$-plane.

3.2. From now on we shall use $p$ to denote the center of the sheet $W_{0}$. Given an arbitrary point $q$ on the surface we can join it to $p$ by a path $Q$ as described in 2.5. We shall denote the length of $Q$ by $L=L(q)$. We wish to show that $Q$ is the shortest path joining $q$ to $p$.

We note first of all that if $g$ is a point of $W_{0}$, then $Q$ is the straight line segment joining $q$ to $p$, and any other curve on the surface joining $q$ to $p$ will have a projection in the $w$-plane longer than $Q$. We proceed by induction, assuming the statement is true for all points on sheets of the first $n-1$ generations, and proving it for points on sheets of the $n$th generation.

Let $q$ be a point on a sheet $H_{n}$ of the $n$th generation, and let the projection of $q$ in the $w$-plane be $w$. Let the fundamental slit of $H_{n}$ be $L$, with end point $c$. Then $H_{n}$ is one of a finite number of sheets of the $n$th generation, each of whose fundamental slit has the same projection as $L$ and the same end point $c$. If $\gamma$ is an arbitrary curve joining $g$ to $p$ it must cross one of these fundamental slits at a point $q_{1}$ which is simultaneously a point on a slit $L_{1}$ of a sheet $H_{k}$ of a generation $k<n$. Then by induction, the part of $\gamma$ from $q_{1}$ to $p$ is at 
least equal in length to the path $Q_{1}$, which starts with the straight line segment on $H_{k}$ from $q_{1}$ to the center of $H_{k}$. But by radiality of the slit $L_{1}$ with respect to the center of $H_{k}$, and the fact that $c$ lies on $L_{1}$, this line segment passes through the point $c$. Thus if we denote by $w_{1}$ and $w_{2}$ the projections of $g_{1}$ and $c$ respectively, we have $L\left(q_{1}\right)=L(c)+\left|w_{2}-w_{1}\right|$. If we further denote by $C$ the part of the path $Q_{1}$ joining $c$ to $p$, we see that the path $Q$ consists of the straight line segment from $q$ to $c$ followed by the path $C$. Hence $L(q)$ $=L(c)+\left|w_{2}-w\right| \leqq L(c)+\left|w_{2}-w_{1}\right|+\left|w_{1}-w\right|=L\left(q_{1}\right)+\left|w_{1}-w\right|$. But the right-hand side is less than or equal to the length of $\gamma$, because the first term is less than or equal to the length of the part of $\gamma$ from $q_{1}$ to $p$, and the second term is less than or equal to the length of the part of $\gamma$ from $q_{1}$ to $q$ whose projection in the $w$-plane joins $w_{1}$ to $w$.

We can therefore speak of the distance of an arbitrary point $q$ to the point $p$ as the length $L(q)$ of $Q$.

3.3. Given an arbitrary real. number $t$, the set of points on the surface whose distance to $p$ is less than $t$ forms a simply-connected region, by the same reasoning as in 2.5. Its boundary $\Gamma(t)$ will therefore be a connected set consisting of all those points at a distance $t$ from $p$. We wish to investigate more closely the structure of $\Gamma(t)$.

We consider first those points of $W_{0}$ whose distance to $p$ is $t$. If the $w$-coordinate of $p$ is $w_{c}$, then they will be the points of the circle $\left|w-w_{0}\right|=t$. $\Gamma(t)$ can only intersect a finite number of sheets, since otherwise there would be an infinite number of branch points at finite distance. On an arbitrary one of these sheets, if its center $c$ has distance $t_{1}$ from $p$ and has w-coordinate $w_{1}$, then the set of points on the sheet whose distance from $p$ is $t$ will be the points of the circle $\left|w-w_{1}\right|=t-t_{1}$. Thus $\Gamma(t)$ will be a curve consisting of the union of a finite number of these circles.

3.4. We shall now introduce a coordinate system on the surface by means of which every point of the surface is uniquely defined by a single pair of coordinates. To do this we start by fixing an arbitrary curve $C$ from $p$ to the boundary, where we require only that no two points of $C$ shall have the same distance to $p$. For example, any ray from $p$ to infinity lying in the interior of $W_{0}$ would do. If we start at any point $q$ of $C$, it must have some fixed distance $t$ from $p$, and we may follow along $\Gamma(t)$ in the positive direction, i.e., that of increasing argument of $w-w_{0}$ for the part of $\Gamma(t)$ on $W_{0}$. Since $\Gamma(t)$ is connected, we must after traversing it just once arrive back at $q$. We can then assign to each point of $\Gamma(t)$ its distance $s$ from $q$ along the curve $\Gamma(t)$. Every point of the surface is therefore uniquely determined by the coordinates $(t, s)$. Namely, $(t, s)$ is that point on $\Gamma(t)$ which is arrived at by following $\Gamma(t)$ in the positive direction a distance $s$ from the (unique) point $q$ on $C$ which is a distance $t$ from the origin.

We shall make extensive use of these coordinates in Theorem 2 of $\S 6$. 


\section{The embedding theorem.}

LEMMA. Let $L_{1}$ and $L_{2}$ be any two rays with the same end point, but not in the same vertical plane in $x, y, z$-space. Let $\Pi$ be the plane through the common end point of $L_{1}$ and $L_{2}$, parallel to the $x, y$-plane. Let the sector bounded by $L_{1}$ and $L_{2}$ project onto a sector $A$ of $\Pi$. Given an arbitrary sheet other than $W_{0}$ of a Riemann surface of class A, we can embed it isometrically in $x, y, z$-space in such a manner that it projects onto the sector $A$, and that the edge $N$ of the fundamental slit coincides with $L_{1}$ and the edge $M$ coincides with $L_{2}$.

Proof. We shall assume that the edges $L_{1}$ and $L_{2}$ are so labeled that if a ray sweeps out the given sector from $L_{1}$ to $L_{2}$, then its projection will sweep out $A$ in the direction of increasing slope. We then divide sector $A$ into three parts by a pair of rays $R_{1}$ and $R_{2}$ from the vertex, where $R_{2}$ is in the direction of increasing slope from $R_{1}$. Let $\alpha$ be the angle between $L_{1}$ and $R_{1}, \beta$ the angle between $R_{1}$ and $R_{2}$, and $\gamma$ the angle between $R_{2}$ and $L_{2}$. (The angle chosen each time is that of the sector bounded by the given rays which projects into $A$.) Starting from the center of the sheet to be embedded we draw rays $S_{1}, \cdots, S_{9}$ in order of increasing argument as follows. $S_{1}$ and the edge $N$ of the fundamental slit are to bound a sector of angle $\alpha . S_{9}$ and the edge $M$ of the fundamental slit bound a sector of angle $\gamma . S_{2}, \cdots, S_{8}$ divide the sector from $S_{1}$ to $S_{9}$ into eight equal parts. Finally, we divide the sector from $R_{1}$ to $R_{2}$ into eight equal parts by rays $T_{1}$ to $T_{7}$.

The embedding of the sheet is done as follows. The sector bounded by $N$ and $S_{1}$ is placed in $x, y, z$-space in such a manner that $N$ coincides with $L_{1}$, and $S_{1}$ with $R_{1}$. Then the sector between $S_{1}$ and $S_{2}$ is placed so that $S_{1}$ coincides with $R_{1}$ and $S_{2}$ projects onto $T_{1}$. The next sector is then placed so that its edge $S_{2}$ coincides with the position of $S_{2}$ just determined, and its edge $S_{3}$ coincides with $T_{2}$. Succeeding sectors are placed in a similar manner, so that each one projects onto a sector of angle $\beta / 8$. Then the edge $S_{9}$ will coincide with $R_{2}$, and the final sector is placed so that the edge $S_{9}$ coincides with $R_{2}$ and $M$ coincides with $L_{2}$.

This completes the embedding. The only condition that must be fulfilled in order to carry it out is that the eight equal sectors in the sheet must have angles greater than $\beta / 8$. But this can always be guaranteed by choosing $R_{1}$ and $R_{2}$ sufficiently close together.

THEOREM. Let $W$ be a Riemann surface of class A. We assume that the sheets of $W$ have the following additional property. At least one edge of each slit, and both edges of the fundamental slit, have an adjacent sector which does not intersect any other slits on the sheet. Then $W$ can be embedded isometrically as a surface of the form $z=f(x, y)$ where $f(x, y)$ is single-valued and continuous.

Proof. We begin the embedding by placing the sheet $W_{0}$ so that it coincides with the $x, y$-plane. Since each slit has on one side of it a sector of some 
given angle disjoint from all the other slits, if we choose the subsector $S$ of half the angle adjacent to each slit, these subsectors will be mutually disjoint. Let the angle of a given one of the sectors $S$ be $\epsilon$. $S$ is bounded by one edge, say $L$, of a slit, and by another ray $R$. We then rotate $S$ about the ray $R$ until it projects onto a sector of angle $\epsilon / 2$. When this has been done for every sector $S$, the sheet $W_{0}$ is embedded so that no two points lie above the same point of the $x, y$-plane, and so that the whole $x, y$-plane is covered except for at most a countable number of sectors, one for each slit on $W_{0}$, over which succeeding generations will be placed.

The proof is completed by induction. We assume that the approximating surface $W_{n-1}$ has been embedded isometrically, and we adjoin the sheets of the $n$th generation. These sheets will be attached in such a manner that if no two points of $W_{n-1}$ project onto the same point of the $x, y$-plane, and if the boundary of $W_{n-1}$ consists of pairs of rays with common end point, then $W_{n}$ will have the same properties.

We consider an arbitrary component of the boundary of $W_{n-1}$, which consists of a pair of rays $L_{1}$ and $L_{2}$ with common end point $V$. Since no two points of $W_{n-1}$ project onto the same point, $L_{1}$ and $L_{2}$ cannot lie in the same vertical plane, and they must form a sector which projects onto a sector $A$ in the horizontal plane through $V$. We must then adjoin a finite number of sheets in the manner described in 2.3. If there is only one sheet to be adjoined, the embedding may be carried out by means of the lemma. In general, if there are $m$ sheets, we divide the sector $A$ into $m$ equal parts by rays $R_{1}, \cdots, R_{m-1}$. We then apply the lemma to $L_{1}$ and $R_{1}, R_{1}$ and $R_{2}, \cdots, R_{m-1}$ and $L_{2}$, in order to embed the $m$ sheets successively. As in the case of $W_{0}$ we next choose mutually disjoint sectors adjacent to each slit on the sheets just adjoined. Furthermore, if along $L_{1}, R_{1}, R_{2}, \cdots, L_{2}$, identification is to be made only up to a point $p$, then we choose a sector adjacent to the part of the slit past $p$, disjoint from all the other sectors. Each of these sectors is then rotated about the edge opposite the slit until it projects onto half of the original angle. We thus achieve an embedding of $W_{n}$ with the desired properties.

To show that the function $f(x, y)$ describing the final surface is singlevalued we need only point out that any two points of the final surface must be contained in $W_{n}$ for some $n$, and hence cannot project onto the same point of the $x, y$-plane. That $f(x, y)$ is continuous follows from the fact that every point of the surface is either in the interior of a plane sector, on a common edge of two such sectors, or else at a common vertex of a finite number of sectors.

This completes the proof of the theorem. There are still several remarks which can be made about this method of embedding.

4.1. We may note first that the extra hypothesis on the structure of the sheets is certainly satisfied if there are only a finite number of slits on each sheet. This hypothesis is not a necessary one, since the embedding can be carried out in many cases where the sheets are not so constructed. However, 
that some condition on the sheets is needed can be seen by observing that the surfaces of the type constructed in Theorem 2 of $\$ 6$ cannot be embedded by our method.

4.2. As was pointed out in the introduction, the embedding method can be extended to many cases of surfaces not of class A. For instance, it may have logarithmic singularities, it may have a boundary at finite distance, and need not be simply-connected, but only schlichtartig.

4.3. Even in cases where the embedding process cannot be carried out, the general idea of it may be used in order to get a better intuitive picture of the structure of the surface. The author has found it useful to consider successive sheets to be placed in the form of closed-up fans along the slit to which they are attached. This may be done for all surfaces of class A, the extra hypothesis of our theorem being needed in order to allow us to "open up" the fans to form an actual surface.

5. An infinitely-differentiable hyperbolic surface. If a surface in 3-space is given by a function $z=f(x, y)$, where $f(x, y)$ is twice differentiable, then it is known [5] that every point of the surface has a neighborhood which can be mapped conformally onto an open disk in the complex plane. These neighborhoods therefore define a complex analytic structure on the surface, in terms of which we may consider the points of the surface, together with the given mappings, to define an abstract Riemann surface. If the original surface was simply-connected, it must then be conformally equivalent to either the unit disk or the whole plane. The question arose whether every such surface which covered the whole $x, y$-plane must be conformally equivalent to the plane. The following theorem answers that question.

THEOREM. There exists a surface of the form $z=f(x, y)$, where $f(x, y)$ is defined, single-valued, and infinitely-differentiable over the whole $x, y$-plane which can be mapped conformially onto the interior of the unit circle.

Proof. What we must show is that the given surface, when considered as a Riemann surface, is hyperbolic. The proof proceeds in two steps. We start with a particular hyperbolic Riemann surface of class $\mathrm{A}$, and use a method similar to that of the previous section to embed it isometrically as a surface which is infinitely-differentiable everywhere except at the branch points. We then show that this surface can be deformed in a sufficiently small neighborhood of every branch point, so that it becomes everywhere infinitely-differentiable while still remaining hyperbolic.

The surface we shall use is the following. We consider the points $x_{n}$, $n= \pm 1, \pm 2, \cdots$ on the real axis, where $x_{n}=n$ if $n<0, x_{n}=2^{n}$ if $n>0$. The surface has a simple branch point over the points $x_{n}$, and is formed by attaching sheets $H_{n}$ to a fixed sheet $H_{0}$.

The sheet $H_{0}$ consists of a copy of the $w$-plane with slits $R_{n}$, where each slit $R_{n}$ projects onto a ray from $x_{n}$ to infinity lying in the upper half plane and 
radial with respect to the point $-i$. The sheet $H_{n}$ has the single slit $R_{n}$ and is attached to $H_{0}$ by crosswise identification over this slit. We thus obtain a surface of class A which belongs to a class of surfaces proved by Potyagailo [10] to be hyperbolic.

In order to make the desired representation of this surface we use the following lemma.

LemMa 1. Given real numbers $a, b$, such that $0<a \leqq b \leqq 2 \pi$, then one can construct an infinitely differentiable surface $S$ in $x, y, z$-space such that $S$ is the isometric image of a plane sector of angle $b$, the boundary of $S$ coincides with the boundary of a sector $T$ in the $x, y$-plane of angle a, and there is exactly one point of $S$ over each point of $T$.

Proof. Introduce polar coordinates $(r, \vartheta)$ in the $x, y$-plane, using the vertex of the sector $T$ as the origin. Then the points of $T$ are given by $\vartheta_{1} \leqq \vartheta \leqq \vartheta_{1}+a$. We shall define an infinitely-differentiable function $h(\vartheta)$ in this interval such that $0 \leqq h(\vartheta) \leqq 1$, and $h\left(\vartheta_{1}\right)=h\left(\vartheta_{1}+a\right)=0$.

For $S$ we shall use a cone whose vertex coincides with that of $T$, and whose generators are given by $z=r \tan h(\vartheta)$ for each $\vartheta$. Thus $z$ will be an infinitely differentiable function of $r, \vartheta$ and hence of $x, y$. Finally, we shall arrange it so that the generator of $S$ sweeps out a total angle of $b$ as $\vartheta$ varies from $\vartheta_{1}$ to $\vartheta_{1}+a$.

To do this we first introduce the function $g(x)=e^{-1 / x} e^{1 /(x-1)}, 0 \leqq x \leqq 1$, where we set $g(0)=g(1)=0$. Then $g(x)$ is infinitely differentiable, and all its derivatives are 0 at $x=0$ and $x=1$. (Derivatives being taken from inside the interval.) Furthermore, looking at the first derivative, one sees immediately that $g(x)$ is increasing for $0<x<1 / 2$, and decreasing for $1 / 2<x<1$. Its maximum value is $g(1 / 2)=e^{-4}$.

We now consider the unit sphere around the vertex of the sector $T$. The points of this sphere are given by $(\vartheta, \phi)$, where $\phi$ is the angle with the horizontal. We define the function $\phi=h(\vartheta), \vartheta_{1} \leqq \vartheta \leqq \vartheta_{1}+a$, by $h(\vartheta)=\lambda g(X)$, for $X$ congruent $\bmod 1$ to $\left(\vartheta-\vartheta_{1}\right) n / a$. The parameters $n$ and $\lambda$ will be fixed later, subject to the conditions that $n$ is a positive integer, and $0 \leqq \lambda \leqq 1$. We note that the function $h$ will be essentially $n$ congruent copies of a compressed function $g$ placed over successive $\vartheta$-intervals of width $a / n$.

We now wish to calculate the arc-length of the curve $C: \phi=h(\vartheta)$ on the unit sphere. We have $d s^{2}=\cos ^{2} \phi d \vartheta^{2}+d \phi^{2}$, and hence $|d \phi| \leqq d s \leqq|d \vartheta|+|d \phi|$. Since $\phi$ goes from 0 to $\lambda e^{-4}$ and then back to 0 in each interval $((m-1) / n) a$ $\leqq \vartheta-\vartheta_{1} \leqq(m / n) a, m=1, \cdots, n$, we have $\int_{C} d s \geqq \int_{C}|d \phi| \geqq 2 n \lambda e^{-4}$.

We now fix $n$ so that $2 n e^{-4} \geqq b$, where $b$ was the quantity given in the hypothesis. For this value of $n$ the length of the curve $C$ will depend only on $\lambda$ and we shall denote it by $L(\lambda)$. When $\lambda=0$ we have $\phi=h(\vartheta) \equiv 0$, and $d s=d \vartheta$, so that $L(0)=\int_{c} d \vartheta=a \leqq b$. On the other hand, $L(1) \geqq 2 n e^{-4} \geqq b$. Hence, since $L$ depends continuously on $\lambda$ we can find a value of $\lambda$ for which $L(\lambda)=b$. 
With $n$ and $\lambda$ so chosen, we form the conic surface $S$ with vertex at the center of the unit sphere, and generated by the curve $C$ on the sphere. Then the total angle of $S$ at the vertex will exactly equal the total length of $C$ which is $b$. Furthermore, we have one generator of $S$ lying over every ray $\vartheta=$ constant for $\vartheta_{1} \leqq \vartheta \leqq \vartheta_{1}+a$, and $S$ therefore satisfies the requirements of the lemma.

We now return to our surface $W$ and complete the construction as follows. From each point $x_{n}$ we draw a ray $R_{n}^{\prime}$ parallel to $R_{n+1}$ if $n>0$, and parallel to $R_{n-1}$ if $n<0$. Let $R_{n}^{\prime \prime}$ be the angle bisector of the sector between $R_{n}^{\prime}$ and $R_{n}$. Then by the lemma, we can embed the sector between $R_{n}^{\prime}$ and $R_{n}$ so that it projects onto the sector between $R_{n}^{\prime}$ and $R_{n}^{\prime \prime}$, keeping the edge $R_{n}^{\prime}$ fixed, and making the other edge coincide with $R_{n}^{\prime \prime}$. We can then (again by the lemma) embed the sheet $H_{n}$ isometrically so that it projects onto the remaining sector between $R_{n}^{\prime \prime}$ and $R_{n}$, and such that the edges to be identified with the upper and lower edges of the slit $R_{n}$ now coincide with $R_{n}$ and $R_{n}^{\prime \prime}$ respectively.

If this is done for each $n$, we obtain an isometric image of the surface $W$ as a surface $S$ in 3-space which covers every point of the $x, y$-plane exactly once. Furthermore, if $S$ is given by $z=f(x, y)$, then $f$ is by construction infinitely-differentiable everywhere, except possibly along the rays $R_{n}, R_{n}^{\prime}$, and $R_{n}^{\prime \prime}$. However, at each point of these rays all derivatives are zero, and $f$ is therefore everywhere infinitely-differentiable except at the points $x_{n}$.

This completes the first step in the proof. We have now a one-one isometric correspondence between $S$ and the surface $W$. We shall denote this correspondence by $W=\Phi(S)$. Furthermore, since $W$ is hyperbolic, there is a one-one conformal mapping $\Psi$ of $W$ onto the interior of the unit circle. The composed mapping $\Psi \Phi$ therefore defines a one-one correspondence between $S$ and the interior of the unit circle which preserves angles everywhere except at the branch points, i.e., the points $x_{n}$.

We now observe that if we make a small circle in the $x, y$-plane around each of the $x_{n}$, then by the Whitney extension theorem [13], we can construct a function $f_{1}(x, y)$ which is everywhere infinitely-differentiable, and which is equal to $f$ in the exterior of these circles.

Let $S_{1}$ be the surface $z=f_{1}(x, y)$. Being everywhere infinitely-differentiable, angles are uniquely defined on $S_{1}$ from its embedding, and we can define a conformal structure on it using these angles. $S_{1}$ then becomes a Riemann surface, and by virtue of the topological projection map onto the whole plane, it is simply-connected. We wish now to show that the surface $S_{1}$ must be hyperbolic provided the circles around the $x_{n}$ are chosen sufficiently small. We proceed as follows.

Let $\zeta_{n}=\Psi\left(\Phi\left(x_{n}\right)\right)$ be the images in the unit circle $|\zeta|<1$ of the points $x_{n}$ on $S$. Since there are only a finite number of these in any compact subset $|\zeta| \leqq r<1$, we may choose circles around each $\zeta_{n}$ such that the sum of the angles subtended at the origin by these circles is less than $\pi$. Corresponding 
to each of these circles is a region $R_{n}$ of $S$. Choose the circles $C_{n}$ around each $x_{n}$ such that the projection on the $x, y$-plane of $R_{n}$ includes the interior of $C_{n}$. We choose $f_{1}$ equal to $f$ outside the $C_{n}$ and show that for this choice, the corresponding surface $S_{1}$ will be hyperbolic. To do this we use the following lemma.

Lemma 2. Let $W$ be a simply-connected Riemann surface. Let $\gamma_{0}$ be an arbitrary simple closed curve on $W$, and let $H$ be the set of all simple closed curves separating $\gamma_{0}$ from the boundary. $\gamma_{0}$ will bound a simply-connected region $\Omega$ on $W$ whose complement we denote by $D$. Given an arbitrary metric $\rho$ on $W$, we denote by $A_{\rho}(D)=\iint_{D} \rho^{2} d x d y$ the area in $\rho$ of $D$, and by $L_{\rho}(\gamma)=\int_{\gamma} \rho d s$ the length in $\rho$ of the curve $\gamma$.

Then a necessary and sufficient condition for $W$ to be hyperbolic is that there exist a metric $\rho$ and an $\epsilon>0$, such that $A_{\rho}(D)$ is finite, and $L_{\rho}(\gamma)>\epsilon$ for all $\gamma$ in $H$.

(Note: the metric $\rho$ is given relative to a fixed coordinate system, and changes with the coordinates in such a manner that the above expressions remain invariant.)

Proof. To see the necessity, assume that $W$ is hyperbolic, and map it conformally onto the unit disk in the $z$-plane in such a manner that some point of $\Omega$ maps into the origin. Then the image of $\Omega$ will include some circle $|z|$ $=R>0$. Let $\rho$ be the metric corresponding to the Euclidean metric in the $z$-plane. Then the area in $\rho$ of $D$ is less than $\pi$, and the length in $\rho$ of each $\gamma$ in $H$ is greater than $2 \pi R$.

As for sufficiency, we show that if the length of all $\gamma$ in $H$ is bounded below by $\epsilon>0$ in a given metric, then either $W$ must be hyperbolic, or else the area of $D$ is infinite. Namely, if $W$ is parabolic we can map it onto the $z$-plane, denoting the image of $\gamma_{0}$ by $\Gamma_{0}$. Let $\rho$ be the metric in the $z$-plane corresponding to the given metric on the surface. Since $\Gamma_{0}$ is compact, it is included in the interior of some circle $|z|=r_{0}$. Then for all $r>r_{0}$, the circle $|z|=r$ corresponds to a curve $\gamma$ of $H$, and hence its length in $\rho$ is greater than $\epsilon$, i.e.,

$$
\begin{aligned}
\epsilon & \leqq \int_{|z|=r} \rho d s=\int_{0}^{2 \pi} \rho r d \vartheta \\
\epsilon^{2} & \leqq \int_{0}^{2 \pi} r d \vartheta \int_{0}^{2 \pi} \rho^{2} r d \vartheta=2 \pi r \int_{0}^{2 \pi} \rho^{2} r d \vartheta \\
\frac{\epsilon^{2}}{2 \pi} \int_{r_{0}}^{r_{1}} \frac{d r}{r} & \leqq \int_{r_{0}}^{r_{1}} \int_{0}^{2 \pi} \rho^{2} r d r d \vartheta .
\end{aligned}
$$

But the right-hand side is just the area in $\rho$ of the annulus $r_{0}<|z|<r_{1}$, and it is bounded below by the left-hand side which tends to infinity as $r_{1}$ tends 
to infinity. Since this annulus corresponds to a subregion of $D$, the area of $D$ must be infinite, which proves the lemma.

We now complete the proof by constructing on $S_{1}$ a metric $\rho$ which satisfies the requirements of the lemma. We define $\rho=0$ for points where $f_{1} \neq f$. Where $f_{1}=f$, so that $S_{1}$ coincides with the surface $S$, it is defined by the induced metric of the unit disk via $\zeta=\Psi \Phi(S)$.

Let $R_{0}$ be the part of $S$ corresponding to $|\zeta| \leqq 1 / 2$, and $S_{0}$ the part of $S_{1}$ lying over the same portion of the $x, y$-plane. Then the area in $\rho$ of $S_{1}-S_{0}$, being less than or equal to the area of the corresponding part $1 / 2<|\zeta|<1$ of the unit disk, is finite. Thus, all that remains to prove is that the lengths in $\rho$ of all curves $\gamma$ separating $S_{0}$ from the boundary are bounded uniformly below by a positive number.

Let $\gamma$ be any such curve, and let $\gamma_{1}$ be its projection on $S$. By the definition of $\rho$, the length of $\gamma$ is equal to the length of the part of $\gamma$ which coincides with $\gamma_{1}$, and this in turn is equal to the length of the image of this part in the unit disk. But $\gamma_{1}$ maps onto a curve $C$ which separates $|\zeta|=1 / 2$ from $|\zeta|=1$. Thus, if $\zeta=r e^{i \phi}$, then $\phi$ takes on all values from 0 to $2 \pi$ along $C$, and $r>1 / 2$. Denote by $E$ the set of values of $\phi$ which are not included in the angles subtended by the circles previously drawn about the points $\zeta_{n}$. Let $D$ be the part of $C$ for which $\phi$ is in $E$. Then the image of $D$ on $S$ is included in the part of $\gamma_{1}$ which coincides with $\gamma$. Combining all these facts, we have for the length in $\rho$ of $\gamma$,

$$
L_{\rho}(\gamma) \geqq \int_{D}|d \zeta|=\int_{E}\left(r^{2}+\left(\frac{d r}{d \phi}\right)^{2}\right)^{1 / 2} d \phi \geqq \int_{E} r d \phi>\frac{1}{2} \int_{E} d \phi \geqq \frac{\pi}{2} .
$$

6. The relation of branching to type. In this section we study the degree to which the type of a surface of class $A$ is determined by the amount of branching, in so far as the branching is measured by the counting function $n(t)$.

6.1. Given a fixed point $p$ on a Riemann surface of class A, we define $n(t)$ to be the number of branch points, including multiplicity, whose distance from $p$ is less than $t$. (See $\S 3$ for the definition of the distance.) The function $n(t)$ was introduced by Ahlfors [1] who proved that a sufficient condition for parabolic type is

$$
\int^{\infty} \frac{d t}{\operatorname{tn}(t)}=\infty
$$

This shows in particular that those surfaces of class A which are so weakly branched that

$$
n(t) \leqq \log t
$$

must all be parabolic. 
We first prove a similar theorem in which a weaker condition is proved sufficient for parabolicity within a special subclass of surfaces of class A.

THEOREM 1. Given a surface of class A which satisfies the additional condition that all its branch points project onto a bounded set of the w-plane, then it will be parabolic provided that

$$
\int^{\infty} \frac{d t}{n(t)}=\infty
$$

or in particular, if

$$
n(t) \leqq t \log t \text {. }
$$

Proof. We apply a theorem of Ahlfors [2] which generalizes his previous result. Namely, in order to prove a surface parabolic, it is sufficient to find a metric $\rho$ on the surface such that if $\Gamma(t)$ is the curve all of whose points are a $\rho$-distance $t$ from a fixed point of the surface, and $L_{\rho}(t)$ is the length of this curve in $\rho$, then

$$
\int^{\infty} \frac{d t}{L_{\rho}(t)}=\infty
$$

The only condition on $\rho$ is that there should be no boundary points at finite distance.

To derive condition (1) for parabolic type, one need only choose the Euclidean metric of the surface. In our case we choose the metric

$$
\rho=\min \left\{1, \frac{R}{|w|}\right\},
$$

where it is assumed that the projections of all the branch points lie in the circle $|w|<R$. If we denote by $r_{m}$ the $\rho$-distance from the origin to the branch point $a_{m}$, then the curve $\Gamma(t)$ is the union of a finite number of $\rho$-circles $C_{m}$, $m=1,2, \cdots, n(t)$, where $C_{m}$ consists of those points of $\Gamma(t)$ which lie in a single sheet and have constant $\rho$-distance $t-r_{m}$ from $a_{m}$.

To find the length of $\Gamma(t)$ we consider an arbitrary one of the $C_{m}$, say $C$. It lies in a single sheet of the surface, and hence may be considered as a plane curve, all of whose points have a fixed $\rho$-distance from some point $w_{0},\left|w_{0}\right|<R$. We may assume without loss of generality that $w_{0}$ is real and positive, i.e., that $w_{0}=s \geqq 0$. We consider first the case that $C$ lies entirely outside of $|w|=R$.

Let $-k_{1}$ be the point where $C$ intersects the negative real axis, and $k_{2}$ be the point where it intersects the positive real axis. In order to evaluate the length of $C$ we must first show that if the point $w=r e^{i v}$ traverses $C, r$ will decrease monotonically from $k_{2}$ to $k_{1}$ as $|\vartheta|$ goes from 0 to $\pi$. This is equivalent to 
showing that as a point traverses $|w|=r>R$ from right to left, its $\rho$-distance to $s$ increases. But suppose $w_{1}=r e^{i \vartheta_{1}}$, and $w_{2}=r e^{i \vartheta_{2}}$, where $\left|\vartheta_{1}\right|<\left|\vartheta_{2}\right| \leqq \pi$. If $g_{i}$ is the geodesic from $z_{i}$ to $s$, then the part of $g_{i}$ inside the circle $|w| \leqq R$ will be a straight-line segment, while the part outside will correspond to a straightline segment $S_{i}$ in the plane of $\log w$. Examining the situation in this latter plane we see that if $S_{2}$ has greater slope than $S_{1}$, then both parts of $g_{2}$ are longer than the corresponding parts of $g_{1}$ and the statement is clearly true. On the other hand, if $S_{1}$ has greater slope than $S_{2}$ we draw an auxiliary curve from $\log w_{1}$ to $\log s$, starting with a straight-line segment $S_{3}$ parallel to $S_{2}$, and then followed by the image $T_{3}$ of a straight-line segment in the $w$-plane. The length of this curve will be at least as great as that of $g_{1}$ by the assumption that the latter was a geodesic, but it will be less than that of $g_{2}$, since $S_{2}$ and $S_{3}$ have equal length, while $T_{3}$ is shorter than the corresponding part of $g_{2}$.

What we have shown then, is that the total variation of $r$ around the curve $C$ is just $2\left(k_{2}-k_{1}\right)$. We therefore have for the length $L$ of $C$,

$$
\begin{aligned}
L= & \int_{C} \rho|d w|=\int_{C} \frac{R|d w|}{|w|}<\frac{R}{k_{1}} \int_{C}(|d r|+r|d \vartheta|) \\
& <\frac{R}{k_{1}}\left(2\left(k_{2}-k_{1}\right)+2 \pi k_{2}\right)<2(\pi+1) R \frac{k_{2}}{k_{1}} .
\end{aligned}
$$

Thus the length of such a curve depends not on the actual values of $k_{1}$ and $k_{2}$, but only on their ratio. However, this ratio can easily be estimated. Let $\tau$ be the $\rho$-distance from $s$ to points of $C$. Choosing paths along the real axis from $-k_{1}$ and $k_{2}$ to $s$, and using the fact that the latter is a geodesic, we have $\tau \leqq R+s+\left(\log k_{1}-\log R\right), \tau=R-s+\left(\log k_{2}-\log R\right)$, and hence $\log k_{2}-\log k_{1}$ $\leqq 2 s<2 R$, or $k_{2} / k_{1}<e^{2 R}$.

Hence we have for all the curves $C_{m}$ that $L_{\rho}\left(C_{m}\right)<2(\pi+1) R e^{2 R}=K$. We have actually only proved this in the case that the projection of $C$ lies outside the $|w|=R$. However, if it lies entirely inside $|w|=R$, then $C$ is a Euclidean circle for which $L_{\rho}(C)<2 \pi R$, and if it intersects $|w|=R$, then by considering separately the parts lying inside and outside, one obtains as above a uniform bound $K$ on its total length. Thus,

$$
L_{\rho}(t)=\sum_{m=1}^{n(t)} L_{\rho}\left(C_{m}\right)<K n(t)
$$

and

$$
\int^{t} \frac{d t}{L_{\nu}(t)}>\int^{t} \frac{d t}{K n(t)}
$$

so that $\left(1^{\prime}\right)$ implies (3) and hence the surface is of parabolic type.

6.2. A simple example of a parabolic surface of class A which satisfies the 
criterion of this theorem and not that of Ahlfors' original theorem is the surface of $\operatorname{arc} \sin w$. Here the branch points all lie above +1 and -1 , and $n(t) \sim t+1$.

Our next object is to show that except by restricting ourselves to special subclasses of surfaces as in the previous theorem, we cannot improve upon Ahlfors' original criterion. The following theorem shows that if we consider all surfaces of class $A$, then condition (1) is the best possible.

THEOREM 2. There exists a subclass of surfaces of class A containing surfaces of both hyperbolic and parabolic type for which the divergence of $\int^{\infty} d t / \operatorname{tn}(t)$ is both necessary and sufficient for parabolic type.

Proof. We use the fact that the type of a simply-connected Riemann surface is invariant under quasiconformal mapping [11]. Our method will be to construct a topological mapping of an arbitrary surface of class $\mathrm{A}$ onto a surface whose type is known, and then investigate under what conditions the given map will be quasiconformal.

Let $W$ be a surface of class A. Let $L(t)$ be the length of the curve $\Gamma(t)$ defined in 3.3. Let

$$
\int_{1}^{\infty} \frac{d t}{L(t)}=R \leqq \infty .
$$

We map $W$ onto the strip $S:-\infty<x<R, 0 \leqq y \leqq 1$ by assigning to the point of $W$ with coordinates $(t, s)$ (see 3.4) the point $(x, y)$, where

$$
x=\int_{1}^{t} d t / L(t), \quad y=s / L(t) .
$$

This maps each curve $\Gamma(t)$ onto a vertical line segment whose top and bottom points are identified. The curve $C$ maps onto the parts of $y=0$ and $y=1$ to the left of $x=R$. The point $p$ corresponds to $-\infty$. By virtue of the map $e^{2 \pi z}$ we see that the strip $S$ is hyperbolic or parabolic according as $R$ is finite or infinite. But as is shown in [1], the integral in (1) converges and diverges simultaneously with $\int^{\infty} d t / \operatorname{tn}(t)$. Our problem is thus reduced to finding a class of surfaces for which the auxiliary map (2) is quasiconformal. We shall use surfaces constructed as follows, denoting them as surfaces of class B.

Let $\left\{r_{n}\right\}$ be an arbitrary increasing sequence of positive numbers. We denote by $w_{n, k}$ the points in the complex $w$-plane defined by $\left|w_{n, k}\right|=r_{n}$,

$$
\begin{aligned}
\arg w_{n, k}=(2 k-1) \pi / 2^{n}, & k=1, \cdots, 2^{n} \text { if } n>1, \\
\arg w_{1, k}=(k-1) \pi / 2, & k=1,2,3,4 .
\end{aligned}
$$

The initial sheet $W_{0}$ of the surface will be the $w$-plane with center at the origin and radial slits from each point $w_{n, k}$ to infinity. To each $w_{n, k}$ we associate a copy $W_{n, k}$ of the $w$-plane with a single radial slit from $w_{n, k}$ to infinity. 
Adjoining each $W_{n, k}$ to $W_{0}$ by crosswise identification over the common slit produces a surface $W$ of class A with only first order branch points over each point $w_{n, k}$. One can obviously make the order of branching arbitrarily large or small by suitable choice of the sequence $r_{n}$.

In order to map this surface onto the strip $S$, we choose for the point $p$ the origin of the sheet $W_{0}$. For $C$ we choose the polygonal ray going first from 0 to $r_{1}$ along the real axis of $W_{0}$, and then from $r_{1}$ to $-\infty$ along the real axis of $W_{1,1}$.

To compute the dilatation we introduce the following notation.

$n(r)=$ number of branch points for which $\left|w_{n, k}\right| \leqq r$,

$n_{\vartheta}(r)=$ number of branch points for which $\left|w_{n, k}\right| \leqq r, 0<\arg w_{n, k} \leqq \vartheta$,

(3) $L_{v}(r)=$ contribution to $L(r)$ coming from sheets $W_{n, k}$ for which

$$
0<\arg w_{n, k} \leqq \vartheta
$$

We must consider separately the cases of points on $W_{0}$ and points on other sheets.

CASE 1. Dilatation at a point of $W_{0}$. Let $w=r e^{\imath \vartheta}$ be an arbitrary point on $W_{0}$. Then its $(t, s)$-coordinates will be given by

$$
t=r, \quad s=r \vartheta+L_{\vartheta}(r)+\pi\left(r-r_{1}\right),
$$

where the first term in the expression for $s$ comes from the part of $L(r)$ lying on $W_{0}$, and the last from the part on $W_{1,1}$. Then by (2), we have

$$
x=\int_{1}^{r} \frac{d t}{L(t)}, \quad y=\frac{r \vartheta+L_{\vartheta}(r)+\pi\left(r-r_{1}\right)}{L(r)},
$$

and $x_{r}=1 / L(r), x_{\vartheta}=0, y_{\vartheta}=r / L(r)$. This last is true because if $r$ is fixed and $\vartheta$ varies sufficiently little, then $L_{v}(r)$ remains constant.

To calculate the dilatation we obtain rectilinear coordinates by setting $u=\log r, v=\vartheta$. Then $x_{u}=y_{v}=r / L(r), x_{v}=0$. Hence we may compute the dilatation $d$ as in [4].

$$
\begin{aligned}
& p=x_{u}+\frac{i}{2} y_{u}, \\
& q=\frac{i}{2} y_{u},
\end{aligned}
$$

and

$$
d=\frac{|p|+|q|}{|p|-|q|}=1+\frac{1}{2}\left|y_{u} / x_{u}\right|^{2}+\left|y_{u} / x_{u}\right|\left(1+\frac{1}{4}\left|y_{u} / x_{u}\right|^{2}\right)^{1 / 2} .
$$

This gives us the dilatation in terms of the $(u, v)$-coordinates, but since 
the mapping $r, \vartheta \rightarrow u, v$ was conformal, it is also the dilatation in the $w$-plane. Thus we see that the boundedness of the dilatation is equivalent to the boundedness of the quantity $\left|y_{u} / x_{u}\right|$. But if $y=f(x)$ is the equation of the image in $S$ of the line $v=$ constant, i.e., of the ray $\vartheta=$ constant, then $y_{u} / x_{u}$ $=f^{\prime}(x)=d y / d x$. We turn now to the computation of this quantity.

We note first that we can write explicitly $n_{\vartheta}(r)=[\vartheta n(r) / 2 \pi]$, where the brackets denote the greatest integer contained in the enclosed expression. To see that this is true, we note that $n_{\vartheta}(r)$ is equal to the number of slits on $W_{0}$ which intersect $|w|=r$ between 0 and $\vartheta$. But the total number of slits intersecting $|w|=r$ is $n(r)$, and these are distributed at equal intervals. In fact, if $r_{m} \leqq r<r_{m+1}$, there will be a slit at $k \pi / 2^{m}$ for $k=1, \cdots, 2^{m+1}$.

Hence we have the inequality

$$
\frac{\vartheta}{2 \pi} n(r)-1<n_{\vartheta}(r) \leqq \frac{\vartheta}{2 \pi} n(r) .
$$

Suppose now we denote by $\sum^{\prime}$ the summation taken over all branch points $w_{n, k}$ for which $\left|w_{n, k}\right| \leqq r, 0<\arg w_{n, k} \leqq \vartheta$. We then have

$$
\begin{aligned}
L_{\vartheta}(r) & =2 \pi \sum^{\prime}\left(r-\left|w_{n, k}\right|\right)=2 \pi \int_{0}^{r} n_{\vartheta}(t) d t, \\
L(r) & =2 \pi r+2 \pi \int_{0}^{r} n(t) d t,
\end{aligned}
$$

where the first term is the contribution of $W_{0}$ and the second term comes from the other sheets.

We now assume $\vartheta$ to be fixed and use a prime to denote derivatives with respect to $r$. Then by (4) and (7),

$$
s^{\prime}=\vartheta+L_{\vartheta}^{\prime}(r)+\pi=2 \pi\left(\frac{\vartheta}{2 \pi}+n_{\vartheta}(r)+\frac{1}{2}\right),
$$

and by (6),

$$
2 \pi\left(\frac{\vartheta}{2 \pi}(1+n(r))-\frac{1}{2}\right)<s^{\prime} \leqq 2 \pi\left(\frac{\vartheta}{2 \pi}(1+n(r))+\frac{1}{2}\right) .
$$

Furthermore, by (7),

$$
L^{\prime}(r)=2 \pi(1+n(r))
$$

Thus,

$$
\frac{\vartheta}{2 \pi}-\frac{1}{2(1+n(r))}<\frac{s^{\prime}}{L^{\prime}} \leqq \frac{\vartheta}{2 \pi}+\frac{1}{2(1+n(r))}
$$

and 


$$
\left|\frac{s^{\prime}}{L^{\prime}}-\frac{\vartheta}{2 \pi}\right| \leqq \frac{1}{2(1+n(r))} .
$$

Similarly, we have by (4), (7), and (6), that

$$
\frac{\vartheta}{2 \pi} L(r)-\pi\left(r+r_{1}\right) \leqq s \leqq \frac{\vartheta}{2 \pi} L(r)+\pi\left(r-r_{1}\right) .
$$

Hence,

$$
\frac{\vartheta}{2 \pi}-\frac{r+r_{1}}{2 L} \leqq \frac{s}{L} \leqq \frac{\vartheta}{2 \pi}+\frac{r-r_{1}}{2 L}
$$

and

(9)

$$
\left|\frac{s}{L}-\frac{\vartheta}{2 \pi}\right| \leqq \frac{2 r}{2 L}=\frac{r}{L} .
$$

Combining (8) and (9), we find

$$
\left|\frac{s^{\prime}}{L^{\prime}}-\frac{s}{L}\right| \leqq\left|\frac{s^{\prime}}{L^{\prime}}-\frac{\vartheta}{2 \pi}\right|+\left|\frac{s}{L}-\frac{\vartheta}{2 \pi}\right| \leqq \frac{1}{2}\left(\frac{1}{1+n(r)}+\frac{2 r}{L}\right) .
$$

But since $y=s / L$, we have

$$
y^{\prime}=\frac{L s^{\prime}-s L^{\prime}}{L^{2}}=\frac{L^{\prime}}{L}\left(\frac{s^{\prime}}{L^{\prime}}-\frac{s}{L}\right) .
$$

Further,

$$
x=\int_{1}^{r} \frac{d t}{L(t)}, \quad x^{\prime}=\frac{1}{L}
$$

and

$$
\frac{d y}{d x}=\frac{y^{\prime}}{x^{\prime}}=L^{\prime}\left(\frac{s^{\prime}}{L^{\prime}}-\frac{s}{L}\right) .
$$

Finally, since $L^{\prime}(r)=2 \pi(1+n(r))$, we have that

$$
\left|\frac{d y}{d x}\right| \leqq \pi(1+n(r))\left(\frac{1}{1+n(r)}+\frac{2 r}{L}\right)<2 \pi\left(1+\frac{r n(r)}{L(r)}\right)
$$

where we have used that $L(r) \geqq 2 \pi r>2 r$.

We may summarize our results so far as follows. The dilatation will be uniformly bounded for points on the sheet $W_{0}$ provided that the distribution of branch points is such that

$$
r n(r) / L(r) \leqq M<\infty .
$$


CASE 2. Dilatation at a point of $W_{n, k}$. We consider now points of an arbitrary but fixed sheet $W_{n, k}$. If $\vartheta=\arg w_{n, k}$, we introduce on $W_{n, k}$ the coordinates

$$
\rho=t-r_{n}, \quad \phi=\arg \left(w-w_{n, k}\right)-\vartheta
$$

where $\arg \left(w-w_{n, k}\right)$ is chosen so that $0 \leqq \phi \leqq 2 \pi$. We then obtain exactly the same expression as previously for the dilatation in terms of the quantity $d y / d x$, where $y=f(x)$ is the equation of the image in $S$ of the ray $\phi=$ constant. Another analogous calculation gives us the inequality for this quantity,

$$
\left|\frac{d y}{d x}\right| \leqq 2 \pi\left(3+\frac{8 \operatorname{tn}(t)}{L(t)}\right)
$$

where the left-hand side is evaluated at the point with coordinates $(t, s)$.

Thus the dilatation will be uniformly bounded over the whole surface provided that condition (11) holds. A somewhat easier condition to work with is that there exist a constant $K$ such that

$$
n(t) \leqq K n(t / 2)
$$

for all $t$.

If this condition is satisfied we have

$$
L(t) / 2 \pi=\int_{0}^{t}(1+n(t)) d t>\int_{t / 2}^{t} n(t) d t \geqq \frac{t}{2} n(t / 2) \geqq \operatorname{tn}(t) / 2 K,
$$

and

$$
\frac{\operatorname{tn}(t)}{L(t)} \leqq \frac{K}{\pi} .
$$

Thus we see that the mapping defined by (2) is quasiconformal for all surfaces of class $B$ which satisfy condition (12). To conclude the proof of our theorem all that remains to show is that among these there are surfaces of both parabolic and hyperbolic type.

We note first that for all surfaces of class $B$, we have that

$$
r_{n} \leqq t<r_{n+1} \Rightarrow n(t)=4+4+8+\cdots+2^{n}=2^{n+1} .
$$

Consequently, if we choose the sequence $r_{n}$ such that

$$
r_{n} \geqq 2 r_{n-1}
$$

for all $n$,

then for $r_{n} \leqq t<r_{n+1}$, we shall have $r_{n-1} \leqq t / 2$, and

$$
n(t)=2^{n+1}=2 n\left(r_{n-1}\right) \leqq 2 n(t / 2) .
$$

Hence (14) implies that (12) holds with $K=2$.

To make $n(t)$ arbitrarily small we need only let $r_{n}$ increase sufficiently rapidly, in which case (14) will be satisfied, so that there is no difficulty in 
obtaining parabolic surfaces. But choosing $r_{n}=2^{n+1}=2 r_{n-1}$ satisfies (14), and for $r_{n} \leqq t<r_{n+1}$, we have by (13), $n(t)=2^{n+1}=r_{n+1} / 2>t / 2$, and

$$
\int^{\infty} \frac{d t}{\operatorname{tn}(t)}<2 \int^{\infty} \frac{d t}{t^{2}}<\infty
$$

so that this surface is hyperbolic.

This concludes the proof of the theorem.

6.3. We may note that the above construction incidentally provides us with examples of hyperbolic surfaces with a much lower order of branching than any previously known. In terms of the function $n(t)$ we may formulate our result as follows.

COROLlaRY. Given an arbitrarily small $\epsilon>0$, there exist hyperbolic surfaces for which $n(t) \leqq t^{*}$.

Proof. Choose $r_{n}=2^{(n+1) / \epsilon}$. Then $r_{n}=2^{1 / \epsilon} r_{n-1}$ and condition (14) is satisfied. But for $r_{n} \leqq t<r_{n+1}$, we have $t<2^{(n+2) / \epsilon}=2^{1 / \epsilon} 2^{(n+1) / \epsilon}$, and by (13), $2 n(t)$ $=2 \cdot 2^{n+1}>t^{\epsilon}$, so that

$$
\int^{\infty} \frac{d t}{\operatorname{tn}(t)}<2 \int^{\infty} \frac{d t}{t^{1+\epsilon}}<\infty,
$$

and this surface is therefore hyperbolic.

On the other hand, for $r_{n} \leqq t<r_{n+1}$, we have $t \geqq 2^{(n+1) / \epsilon}=n(t)^{1 / \bullet}$, and hence, $t^{\epsilon} \geqq n(t)$.

6.4. A natural question to ask is whether one can obtain a decisive criterion for the type of surfaces of class A using only the growth of the function $n(t)$. The answer is no, since by the above corollary there exist hyperbolic surfaces with $n(t)<t^{1 / 2}$, while for the surface of arc $\sin w, n(t)>t$. We might mention that Myrberg claimed to have settled this question in [7]. However, the surfaces he constructs are not of class A since they have interior points over the point at infinity, and the part of the surface lying over the plane is not simply-connected.

In view of this situation one might ask if it were at least possible to get some general criterion for hyperbolic type involving only sufficiently large growth of $n(t)$. Again this turns out to be impossible, as the following example shows.

Let $H_{n}, n=0, \pm 1, \pm 2, \cdots$, be a copy of the $w$-plane slit along the real axis from $-\infty$ to $a_{n}$, and from $b_{n}$ to $+\infty$, where $a_{n}<b_{n}$ and $a_{2 n}=a_{2 n-1}$, $b_{2 n}=b_{2 n+1}$. By crosswise identification over the common slits of successive sheets one obtains a surface $W$ of class A which in the case of $a_{n}=-1$, $b_{n}=+1$, for all $n$, reduces to that of arc $\sin w$.

It has been proved in two different places ([6] and [12]) that all surfaces formed in this way are parabolic. But it is immediately clear that given any 
increasing function $f(t)$, we can choose the lengths of the segments $\left(a_{n}, b_{n}\right)$ to decrease sufficiently rapidly so that $n(t)>f(t)$. Thus there exist parabolic surfaces with $n(t)$ arbitrarily large, and any sufficient condition for hyperbolic type must either be restricted to a special class of surfaces, or else involve an added condition.

\section{BIBLIOGRAPHY}

1. L. Ahlfors, Zur Bestimmung des Typus einer Riemannschen Fläche, Comment. Math. Helv. vol. 3 (1931) pp. 173-177. $30-32$.

2. - Sur le type d'une surface de Riemann, C. R. Acad. Sci. Paris vol. 201 (1935) pp.

3. - Conformal mapping, Lecture Notes at Oklahoma Agricultural and Mechanical College, Summer, 1951. pp. 1-58.

4. - On quasiconformal mappings, Journal d'Analyse Mathématique vol. 3 (1953)

5. L. Lichtenstein, Beweis des Satzes, dass jedes hinreichend kleine, im wesentlichen stetig gekrümmte, singularitätenfreie Flächenstück auf einer Teil einer Ebene zusammenhängend und in den kleinsten Teilen ähnlich abgebildet werden kann, Abhandlungen der Königlichen Preussichen Akademie der Wissenschaften zu Berlin, Physikalish-Mathematische Classe, 1911, Abh. VI, pp. 1-49.

6. G. MacLane, Concerning the uniformization of certain Riemann surfaces allied to the inverse-cosine and inverse-gamma surfaces, Trans. Amer. Math. Soc. vol. 62 (1947) pp. 99-113.

7. P. J. Myrberg, Über die Bestimmung des Typus einer Riemannschen Fläche, Annales Academiae Scientiarum Fennicae Ser. A vol. 45 No. 3 (1935) pp. 1-30.

8. R. Osserman, On a conjecture in the problem of type for simply-connected Riemann surfaces, Proceedings of the International Mathematical Congress, Amsterdam, Sept. 1954, vol. 2, p. 153.

9. - A hyperbolic surface in 3-space, Proc. Amer. Math. Soc. vol. 7 (1956) pp. 54-58.

10. D. B. Potyagailo, Condition of hyperbolicity of a class of Riemann surfaces, UkrainskiY MatematiそeskiY Žurnal vol. 5 (1953) pp. 459-463. [Math. Reviews vol. 15 (1954) p. 787.]

11. O. Teichmüller, Eine Anwendung quasikonformer Abbildungen auf das Typenproblem, Deutsche Mathematik vol. 2 (1937) pp. 321-327.

12. L. I. Volkovyskii, The determination of the type of certain classes of simply connected Riemann surfaces, Mat. Sbornik N. S. vol. 23 (65) (1948) pp. 229-258. [Math. Reviews vol. 10 (1949) p. 364.]

13. H. Whitney, Analytic extensions of differentiable functions defined in closed sets, Trans. Amer. Math. Soc. vol. 36 (1934) pp. 63-89.

HARVARD UNIVERSITY,

Cambridge, Mass. 